\title{
The effect of residual neurological deficit on oral glucose tolerance in persons with chronic spinal cord injury
}

\author{
WA Bauman*,1,2,3, RH Adkins ${ }^{3,4}$, AM Spungen ${ }^{1,2,3}$ and RL Waters ${ }^{3,4,5}$ \\ ${ }^{1}$ Departments of Medicine and Rehabilitation Medicine, Spinal Cord Damage Research Center, Mount Sinai Medical \\ Center, New York, NY, USA; ${ }^{2}$ Spinal Cord Injury Service, Veterans Affairs Medical Center, Bronx, NY, USA; \\ ${ }^{3}$ Rehabilitation Research and Training Center on Aging with Spinal Cord Injury, Rancho Los Amigos Medical Center, \\ Downey, CA, USA; ${ }^{4}$ Regional Spinal Cord System of Southern California, Rancho Los Amigos Medical Center, Los \\ Angeles County, Downey, CA, USA; ${ }^{5}$ University of Southern California Medical Center, Los Angeles, CA, USA
}

Study design: Oral glucose tolerance testing was performed prospectively in 201 subjects with spinal cord injury (SCI). The dependent variables included the values from the oral glucose tolerance test (glucose and insulin) and diagnostic classification (i.e., diabetes mellitus, impaired glucose tolerance, normal glucose tolerance); the independent variables consisted of study group, gender, ethnic group, age, age at onset of SCI, duration of injury, and anthropometric measurements.

Objective: To determine the potential effects of level and completeness of SCI on oral glucose tolerance testing. In addition, the effects of gender ethnicity, age, age at onset of SCI, duration of injury, and anthropometric measurements on glucose tolerance were investigated.

Setting: Subjects with chronic SCI were recruited during their annual physical examination at the Comarr Spinal Injury Clinic at Rancho Los Amigos Medical Center, Downey, California.

Methods: An oral $75 \mathrm{~g}$ glucose load was administered after an overnight fast. Serum glucose was determined by autoanalyzer and plasma insulin levels, by radioimmunoassay. The results are reported as mean plus or minus standard error of the mean (mean $\pm \mathrm{SEM}$ ). Analysis of variance (ANOVA) applying a Scheffe' post hoc $\mathrm{F}$ ratio was used for the continuous variables. Chi-squared analyses were performed to determine differences between the groups and among the subgroups for per cent distribution. Linear regression analyses were performed between variables of interest. Stepwise regression analyses were used to predict peak serum glucose concentration and peak plasma insulin level from potential determinants.

Results: The total group consisted of 169 men with a mean age of $38 \pm 0.80$ (range $=20-73$ ) years and 32 women with a mean age of $44 \pm 2.13$ (range $=20-72$ ) years. The distribution by ethnicity for the total group with SCI consisted of $114(57 \%)$ Latino, $54(27 \%)$ white, and 28$)$ $14 \%$ ) African American individuals. There was no significant difference in ethnic distribution among the subgroups for neurological deficit. Subjects were grouped by tetraplegia (Tetra; $n=81$ ) or paraplegia (Para; $n=120$ ) and by subgroup for degree of neurological deficit: complete Tetra $(n=56)$, incomplete Tetra $(n=25)$, complete Para $(n=84)$, and incomplete Para $(n=36)$. Of the total group, 27 subjects $(13.4 \%)$ had diabetes mellitus and $56(28.8 \%)$ had impaired glucose tolerance. Significantly more subjects in the complete Tetra group were classified with a disorder of carbohydrate metabolism than in the other neurological deficit subgroups $(73$ vs $44 \%, 24 \%$ and $31 \%$, respectively for level of decreasing neurological deficit; $\left.X^{2}=36.9, P<0.0001\right)$. The complete Tetra group had significantly higher serum glucose concentrations at $60 \mathrm{~min}, 90 \mathrm{~min}$, and $120 \mathrm{~min}$ and serum insulin concentration at 90 and 120 min compared with the other neurological subgroups $(P<0.05$ for each time point). No differences for plasma glucose were evident between men and women, however, plasma insulin levels were significantly higher for men at the intermediate time points $(30 \mathrm{~min}, 60 \mathrm{~min}$ and $90 \mathrm{~min}$ ), suggesting a relative state of insulin resistance in men. By stepwise regression analyses, higher peak serum glucose concentrations were associated with increased total body $\%$ fat, highest level of lesion (complete Tetra $v s$ other neurological subgroups), older age at time of injury, and male gender; higher peak plasma insulin was associated with increased

*Correspondence: WA Bauman, Director, Spinal Cord Damage 
total body \% fat and male gender.

Conclusions: This study is the first to report that those individuals with the greatest levels of neurological deficit have increased risk of developing disorders of carbohydrate metabolism. Males with SCI are more insulin resistant than females. Glucose tolerance appears to be independent of the effects of ethnicity.

Sponsorship: Funded in part by the National Institute on Disability and Rehabilitation Research (NIDRR) Grant \# H133B30029. This work would not have been possible without the gracious support of Quest Diagnostics, Inc.

Keywords: paraplegia; tetraplegia; diabetes mellitus; hyperinsulinemia; Syndrome X

\section{Introduction}

It has been appreciated that individuals with spinal cord injury (SCI) have an increased prevalence of diabetes mellitus. In 1994, Bauman et al reported that $22 \%$ of 100 veterans with SCI had diabetes mellitus by the World Health Organization classification, whereas, only $6 \%$ of the able-bodied controls had diabetes mellitus. Relationships between obesity, disorders of carbohydrate metabolism, and increased serum uric acid have been described in the general population. ${ }^{2,3}$ In individuals with SCI, the metabolic abnormalities of glucose intolerance, hyperinsulinemia, depressed HDL-cholesterol, and elevated uric acid tend to cluster together to impart increased risk for coronary heart disease. ${ }^{4,5}$ Cardiovascular disease has been reported to occur at earlier ages in individuals with SCI than in the general population. ${ }^{6-9}$

The degree of immobilization in persons with SCI is closely related to the neurological deficit. Because increasing levels of neurological deficit may potentially predispose to an adverse metabolic profile in a stepwise fashion, as has been recently reported for serum lipids, ${ }^{10}$ we addressed the question as to whether persons with greater neurological deficits are at increased risk of abnormalities in oral carbohydrate handling. In addition, we investigated relationships between metabolic variables (serum glucose concentration, plasma insulin and uric acid levels) and potential determinants (body mass index, total body \% fat, age, duration of injury, ethnicity, and gender) for these variables.

\section{Methods}

Oral glucose tolerance testing was performed during a 48 month period from May 1994 to May 1998, on 201 subjects who were being seen for routine annual physical examination at the Comarr Spinal Injury Clinic at Rancho Los Amigos Medical Center, Downey, California. The subjects were divided into two groups: tetraplegia (Tetra) and paraplegia (Para). These two groups were further divided into four subgroups of varying neurological deficit: complete tetraplegia (Com Tetra), incomplete tetraplegia (Incom Tetra), complete paraplegia (Com Para), and incomplete paraplegia (Incom Para). Completeness of lesion was defined according to ASIA standards. ${ }^{11}$ Ethnicity was determined by subject's response to the question of ethnic background.

After an overnight fast, blood was drawn at baseline and at $30 \mathrm{~min}, 60 \mathrm{~min}, 90 \mathrm{~min}$ and $120 \mathrm{~min}$ after administration of a $75 \mathrm{~g}$ oral glucose load. Blood for glucose assays was collected with sodium fluoride, and for insulin, with heparin. Blood was immediately centrifuged and separated for analysis and frozen $\left(-20^{\circ} \mathrm{C}\right)$ until analysis was performed. Glucose determinations were performed on an automated glucose analyzer (CX3, Beckman Instruments, Brea, CA, USA). Plasma insulin levels were obtained by radioimmunoassay according to methods established in the Berson Research Laboratory. ${ }^{12,13}$ Plasma insulin and glucose values were summed by adding determinations at each time between 0 and $120 \mathrm{~min}$. Criteria for the diagnosis of normal (NL) glucose tolerance, impaired glucose tolerance (IGT), or diabetes mellitus (DM) were those of The Expert Committee on the Diagnosis and Classification of Diabetes Mellitus. ${ }^{14}$ Hyperinsulinemia was defined as a plasma insulin value greater than $150 \mu \mathrm{U} / \mathrm{ml}^{1}$ Protein bound glucose (PBG) was determined on a multichannel autoanalyzer (Olympus, Long Island, NY, USA) by Quest Diagnostics, Inc.

Body mass index (BMI) was calculated [body weight/height squared $\left.\left(\mathrm{kg} / \mathrm{m}^{2}\right)\right]$ for all subjects studied. An estimate of per cent body fat (\% fat) in subjects with SCI was performed using a regression equation to predict \%fat [\%fat (all ethnicities $)=3.929+1.246(\mathrm{BMI}) ; \quad(n=60, \quad R=0.58$, $P<0.0001]$. This regression equation (unpublished) was developed from BMI determinations and dual X-ray absorptiometry measurements of body fat in persons with SCI; a prior study in persons with SCI has suggested that DXA measurement is a good surrogate measurement of body adiposity. ${ }^{15}$

In the morning after an overnight fast, blood samples were collected for serum uric acid determinations. Blood was immediately centrifuged and separated for analysis or frozen $\left(-20^{\circ} \mathrm{C}\right)$ until analysis was performed. Serum uric acid determinations were performed on an multichannel autoanalyzer (Olympus, Long Island, NY, USA) by Quest Diagnostics, Inc.

The results are reported as mean plus or minus standard error of the mean (mean \pm SEM). All 
analyses were performed on a personal computer using the Statview 5.0 (SAS Institute, Inc., Cary, NC, USA) software program. Analysis of variance (ANOVA) applying a Scheffe' post hoc F ratio was used for the continuous variables. Chi-squared analyses were performed to determine differences between the groups and among the subgroups for per cent distribution. Linear regression analyses were performed between the following variables: basal and peak serum glucose and insulin levels; BMI and total body \%fat $\mathrm{BMI} / \%$ fat and metabolic variables; age and \%fat, sum serum glucose, and protein bound glucose. Stepwise regression of BMI, ethnicity, gender, neurological deficit, duration of injury, age, and age at time of injury were used to predict peak serum glucose concentration and peak plasma insulin level.

\section{Results}

The total group consisted mostly of men $(n=169)$ with a mean age of $38.1 \pm 0.80$ (range $=20$ to 73 ) years, and duration of injury (DOI) of $13.3 \pm 0.65$ (range $=1$ to $41)$ years. The 32 women had a mean age of $44.4 \pm 2.13$ (range $=20$ to 72) years, and DOI of $17.9 \pm 0.12$ (range $=2$ to 43 ) years. Men were younger than women $(38 \pm 0.80$ vs $44.4 \pm 2.13$ years, $P<0.005)$ and had a shorter duration of injury $(13.3 \pm 0.65 v s$ $17.9 \pm 2.12, P<0.01)$. The distribution of ethnicity for the total group with SCI consisted of $114(57 \%)$ Latino, $54(27 \%)$ white, and 28 (14\%) African American individuals. Latinos were younger than whites or African Americans $(36.0 \pm 0.90$ vs $44.6 \pm 1.35$ and $41.8 \pm 2.41$ years, respectively;
$P<0.0001)$, and Latinos had the shortest duration of injury $(10.6 \pm 0.75$ vs $20.5 \pm 1.16$ and $15.9 \pm 1.78$ years, respectively; $P<0.0001)$. The characteristics of the subjects partitioned by neurological and gender subgroups are shown (Table 1a and Table 2a). There were no significant differences for distribution of gender in the Comp Tetra group compared with the other neurological subgroups individually or together; as such, between men and women, there was no significant differences for the distribution of neurological subgroups.

In the total group, 27 subjects $(13.4 \%)$ had DM and $56(27.8 \%)$ had IGT. After a standard oral glucose load, the Com Tetra group compared with all other subgroups by neurological deficit had higher values for serum glucose concentration at $60 \mathrm{~min}, 90 \mathrm{~min}$ and $120 \mathrm{~min}$ (Figure 1a) and for plasma insulin at $90 \mathrm{~min}$ and 120 min (Figure 1b). The peak and sum plasma insulin levels were higher in the Com Tetra group compared with the other subgroups (Table 1b). No differences for serum glucose were evident between men and women, however, plasma insulin levels were higher for men at the intermediate time points of $30 \mathrm{~min}, \quad 60 \mathrm{~min}$ and $90 \mathrm{~min}$ (30: $105 \pm 6.18$ vs $72 \pm 10.54 \mathrm{mg} / \mathrm{dl}, \quad P<0.05 ; \quad 60: \quad 139 \pm 8.3 \quad$ vs $90 \pm 13.0 \mathrm{mg} / \mathrm{dl}, \quad P<0.05 ; \quad 90: \quad 137 \pm 9.1 \quad v s$ $79 \pm 10.1 \mathrm{mg} / \mathrm{dl}, P<0.01$, respectively). Peak and sum plasma insulin levels were significantly higher in men than women (Table 2b). There were no significant ethnic group differences found for serum glucose concentration.

Subjects in the Com Tetra group had a significantly higher percentage of classifications of disorders of oral carbohydrate metabolism than the other three groups

Table 1 Neurological deficit vs demographic, anthropometric and metabolic parameters

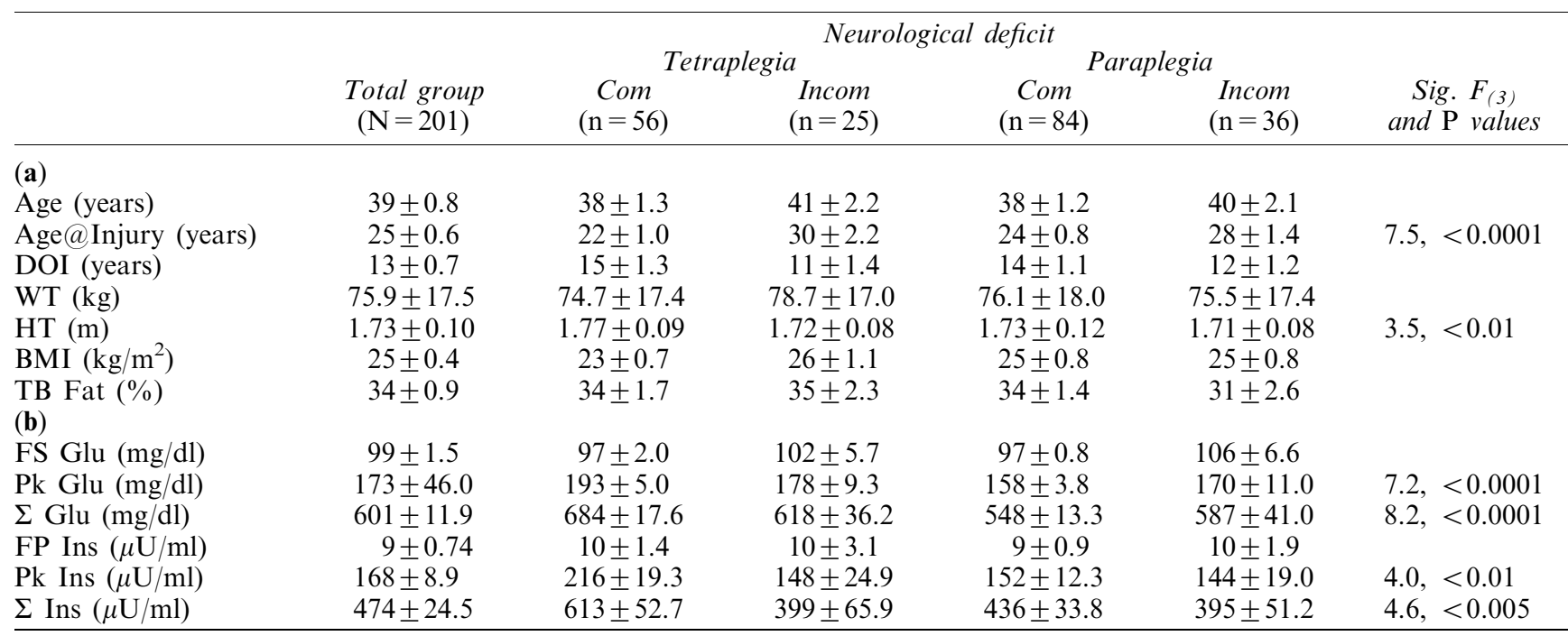

$\mathrm{DOI}=$ duration of injury, $\mathrm{HT}=$ height, $\mathrm{WT}=$ weight, $\mathrm{BMI}=$ body mass index, $\mathrm{TB} \mathrm{Fat}(\%)=$ total body per cent fat. FS $\mathrm{Glu}=$ fasting serum glucose, $\mathrm{Pk}$ Glu $=$ peak serum glucose, $\Sigma \mathrm{Glu}=$ sum of time points $0,30,60$, 90, and 120. FP Ins $=$ fasting plasma insulin, $\mathrm{Pk}$ Ins = peak plasma insulin, $\Sigma \mathrm{Glu}=$ sum of time points $0,30,60$, 90, and 120 . Sig $\mathrm{F}_{(3)}=$ significant $\mathrm{F}$ statistic with 3 degrees of freedom for the four neurological deficit subgroups 
Table 2 Gender $v s$ demographic, antropometric and metabolic parameters

\begin{tabular}{lccc}
\hline & $\begin{array}{c}\text { Total } \\
\text { group } \\
(\mathrm{N}=201)\end{array}$ & $\begin{array}{c}\text { Females } \\
(\mathrm{n}=32)\end{array}$ & $\begin{array}{c}\text { Males } \\
(\mathrm{n}=169)\end{array}$ \\
\hline (a) & & & \\
Age (years) & $39 \pm 0.8$ & $44 \pm 2.1$ & $38 \pm 0.8^{*}$ \\
Age@Injury (years) & $25 \pm 0.6$ & $26 \pm 1.7$ & $24 \pm 0.6$ \\
DOI (years) & $13 \pm 0.7$ & $17 \pm 2.1$ & $13 \pm 0.6 \dagger$ \\
WT (kg) & $75.9 \pm 17.5$ & $70.5 \pm 18.7$ & $77.0 \pm 17.5 \ddagger$ \\
HT (m) & $1.73 \pm 0.10$ & $1.65 \pm 0.07$ & $1.75 \pm 0.10 \dagger$ \\
BMI $\left(\mathrm{kg} / \mathrm{m}^{2}\right)$ & $25 \pm 0.4$ & $25 \pm 1.0$ & $25 \pm 0.5$ \\
TB Fat $(\%)$ & $34 \pm 0.9$ & $44 \pm 2.2$ & $32 \pm 0.9 \ddagger$ \\
$(b)$ & & & \\
FS Glu $(\mathrm{mg} / \mathrm{dl})$ & $99 \pm 1.5$ & $100 \pm 2.2$ & $99 \pm 1.8$ \\
Pk Glu $(\mathrm{mg} / \mathrm{dl})$ & $173 \pm 46.0$ & $171 \pm 8.3$ & $173 \pm 3.5$ \\
$\Sigma$ Glu $(\mathrm{mg} / \mathrm{dl})$ & $601 \pm 11.9$ & $606 \pm 30.7$ & $601 \pm 13.0$ \\
FP Ins $(\mu \mathrm{U} / \mathrm{ml})$ & $9 \pm 0.74$ & $8.75 \pm 1.3$ & $10 \pm 0.9$ \\
Pk Ins $(\mu \mathrm{U} / \mathrm{ml})$ & $168 \pm 8.9$ & $121 \pm 14.1$ & $177 \pm 10.2 \S$ \\
$\Sigma$ Ins $(\mu \mathrm{U} / \mathrm{ml})$ & $474 \pm 24.5$ & $332 \pm 34.9$ & $501 \pm 28.0 \dagger$ \\
\hline
\end{tabular}

Abbreviations for demographic categories may be found in the legend to Table 1. FP Ins=fasting plasma insulin, $\mathrm{Pk}$ Ins $=$ peak plasma insulin, $\Sigma \mathrm{Glu}=$ sum of time points 0,30 , 60, 90, and 120. For females vs males. $\S P<0.05, \uparrow P<0.01$, $* P<0.05, \ddagger P<0.0001$
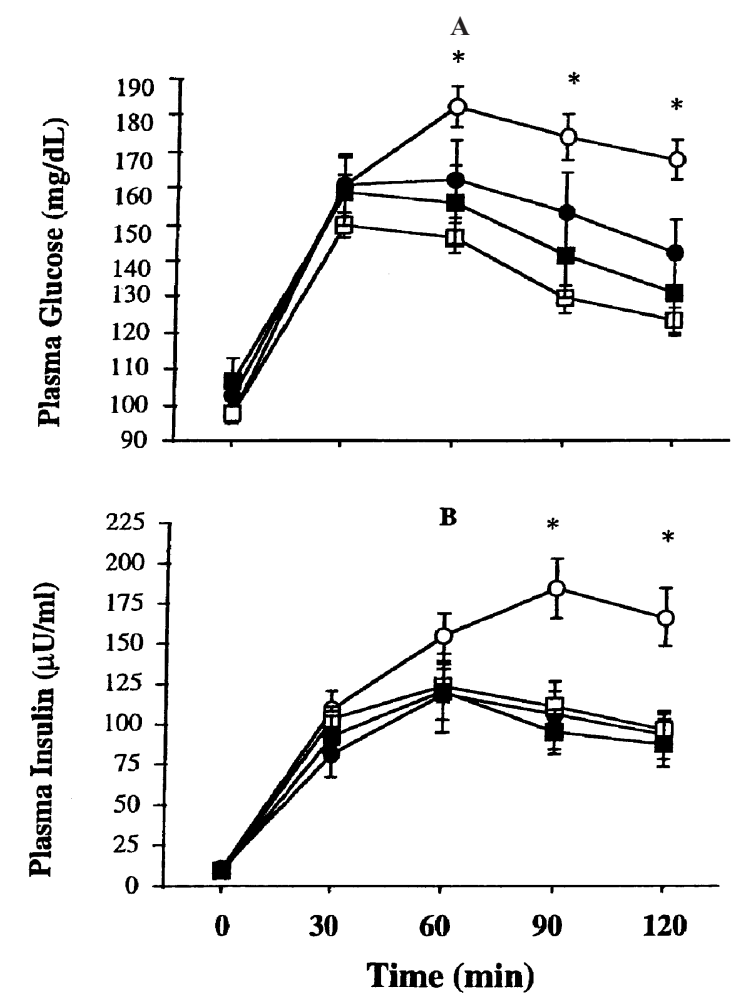

$-\mathrm{O}-$ com tetra

incom tetra

마- com para

incom para

Figure 1 (A) Serum glucose concentration $v s$ time after a $2 \mathrm{~h}$ oral glucose tolerance test; (B) plasma insulin levels $v s$ time after a $2 \mathrm{~h}$ oral glucose tolerance test. Significance $(P<0.05)$ is connoted by an asterisk $(*)$
(73\% vs 44\%, 24\% and $31 \%$, respectively for level of increasing neurological deficit, $\left.X_{(6)}^{2}=36.9, P<0.0001\right)$. From the $2 \mathrm{~h}$ serum glucose value, DM was found with an increased frequency in the Com Tetra group compared with Incom Tetra, Com Para, and Incom Para $(23 \%$ vs $16 \%, 6 \%$ and $11 \%$, respectively). Half of all persons diagnosed with DM from the $2 \mathrm{~h}$ glucose concentration were in the Com Tetra group. Only seven subjects were diagnosed with DM from the fasting serum glucose concentration: Two Com Tetra, one Incom Tetra, and four Incom Para, six of the seven had a $2 \mathrm{~h}$ serum glucose value that was classified as DM. In the Com Tetra group, only $28 \%$ had a normal $2 \mathrm{~h}$ serum glucose concentration compared with $56 \%$ in the Incom Tetra, $79 \%$ in the Com Para, and $69 \%$ in the Incom Para. No significant gender or ethnic differences were found for any of the diagnostic categories.

Hyperinsulinemia was present more frequently in the Tetra group than the Para group (53\% vs $37 \%$, $\left.X^{2}{ }_{(2)}=5.09, P<0.05\right)$. The Com Tetra group had more than half of their members with hyperinsulinemia $(59 \%)$. Although males tended to have greater hyperinsulinemia than females $(46 \%$ vs $31 \%)$, this did not reach significance. Significant differences for ethnic categories were not found for hyperinsulinemia.

In the total group, basal serum glucose concentrations were correlated with peak serum glucose concentrations $(r=0.65, \quad P<0.0001)$. Also, basal plasma insulin concentrations were related to peak plasma insulin concentrations $(r=0.33, P<0.0001)$. BMI was found to correlate with total body \%fat $(r=0.44, P<0.0001)$. BMI and total body $\%$ fat were significantly correlated with basal, peak and sum glucose and insulin values. Age and duration of injury were correlated with \% fat $(r=0.45, P<0.0001$; $r=0.33, P<0.0001)$.

In the total group, age was significantly related to sum serum glucose (Figure 2a) and serum PBG (Figure 2b). Serum PBG was associated with duration of injury in the Incom Tetra and Com Para groups $(P<0.05)$, approaching significance in the Com Tetra group $(P=0.08)$.

Serum uric acid was significantly higher in men than women $(6.3 \pm 0.10$ vs $5.2 \pm 0.54 \mathrm{mg} / \mathrm{dl})$. Uric acid was not significantly different among any of the neurological subgroups, nor by any ethnic classification. However, serum uric acid was significantly related to BMI $(r=0.32, \quad P<0.0001)$, peak serum glucose $(r=0.21, \quad P=0.001)$, and peak plasma insulin $(r=0.16, P=0.01)$.

To determine the significant variables for serum peak glucose or peak plasma insulin values, stepwise regression analyses were performed. Higher peak glucose was most associated with increased total body \%fat, the highest level of lesion (Com Tetra vs other neurological subgroups), older age at time of injury, and male gender. Higher peak insulin was most associated with increased total body \% fat and male gender. 
A
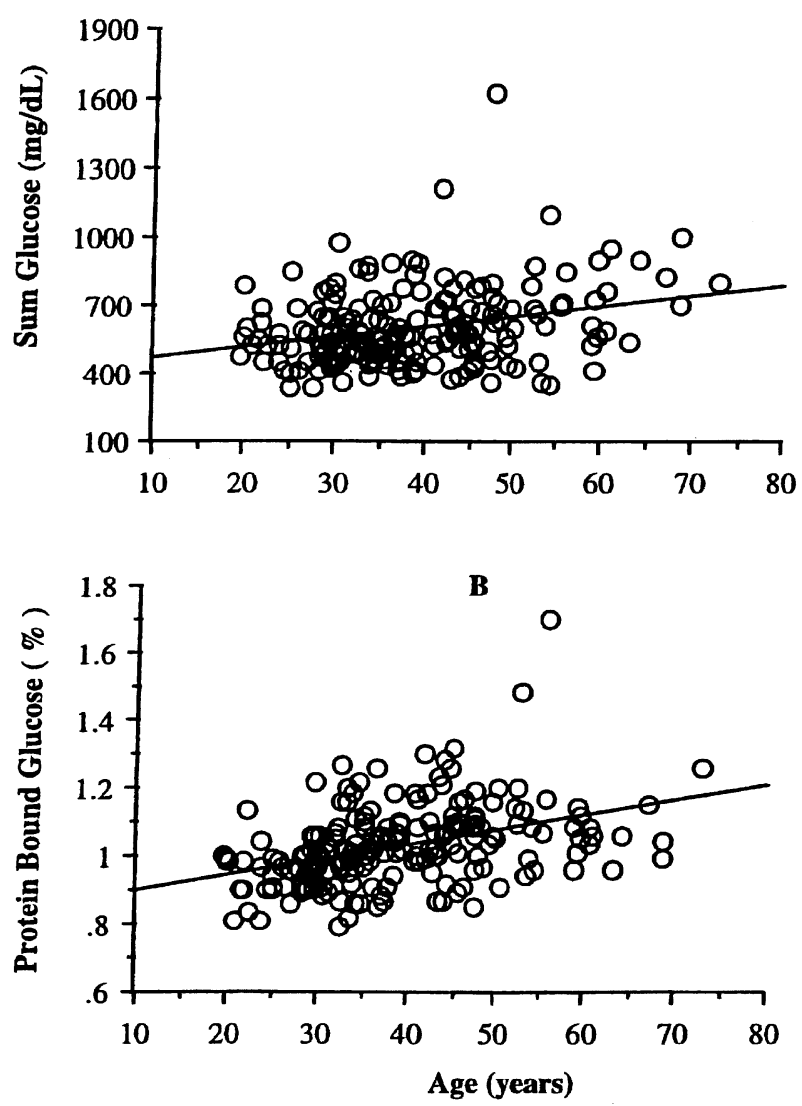

Figure 2 (A) Sum serum glucose concentrations $v s$ age. $(R=0.28, \quad P<0.0001)$; (B) Protein bound glucose $v s$ age $(R=0.40, P<0.0001)$

\section{Discussion}

Disorders of carbohydrate metabolism are more frequent in persons with SCI. In 1980, Duckworth et $a l^{16}$ found that 23 of 45 subjects with chronic SCI had DM. Twelve of these 23 subjects with DM were noted to be hyperinsulinemic and insulin resistant. ${ }^{17}$ Bauman and Spungen ${ }^{1}$ studied 100 veterans with SCI, 50 with paraplegia and 50 with tetraplegia, compared with 50 able-bodied veteran controls. In those with SCI, 22\% had DM by the World Health Organization criteria, ${ }^{17}$ whereas only $6 \%$ of the controls had DM. ${ }^{1}$ The subjects with SCI and DM were generally older, but those with SCI became diabetic at younger ages than did the controls. Individuals with SCI had a significantly higher mean insulin response to an oral glucose challenge than the controls, suggesting insulin resistance. $^{1}$ The investigators did not report a significant effect of the level of neurological deficit noted on these findings. Prolonged inactivity has been demonstrated to result in disorders of glucose tolerance and hyperinsulinism. ${ }^{18}$ In the group with paraplegia, cardiopulmonary fitness $\left(\mathrm{VO}_{2} \max \right)$ was the strongest predictor of insulin resistance. ${ }^{1}$ Thus, it follows that persons with the greatest neurological impairment would be least active (albeit not quantitated in this study) and manifest the highest frequency of abnormalities in carbohydrate tolerance. In the study herein, the group with complete tetraplegia had the worst carbohydrate tolerance, was relatively hyperinsulinemic, and had the highest frequency of DM and glucose intolerance compared with the other neurological deficit subgroups. Of note, the diagnosis of DM would not have been made in 19 of the 27 subjects without provocative testing with a standard glucose challenge. Thus, obtaining a fasting serum glucose value is a poor discriminator of diagnostic classification in persons with SCI. Using stepwise regression analysis, level of lesion (complete tetraplegia compared with other neurological deficit subgroups), age at time of injury and gender were associated with a higher peak serum glucose concentration, while male gender was associated with a higher peak plasma insulin level.

Obesity is associated with insulin resistance, hyperinsulinemia, and carbohydrate disorders. In adult-onset obesity, the size of the fat cell is related to insulin action, ${ }^{19}$ with adipocyte hypertrophy correlated with decreased insulin-mediated glucose uptake. $^{20,21}$ Individuals with SCI have an environmentally-induced form of obesity that usually occurs in early adulthood. In our study by stepwise regression analysis, after an oral glucose load, higher per cent body fat was most strongly associated with higher peak serum glucose and plasma insulin values.

The predominant peripheral tissue responsible for insulin-induced glucose uptake is muscle, and SCI results in an absolute decrease in muscle mass with associated morphological, physiological, and biochemical changes. Thus, a probable etiology of the abnormality in carbohydrate handling noted in subjects with SCI, and especially those with tetraplegia who have the greatest neurological deficit, may result from morphological changes and lean tissue atrophy with associated potential receptor and postreceptor perturbations. It is beyond the scope of this report to address adequately issues of muscle mass and biochemistry, but a recent review has discussed some of these considerations. ${ }^{22}$ Aksnes et $a l^{23}$ studied whole body insulin-induced glucose uptake and isolated denervated muscle glucose transport, as well as other biochemical parameters of muscle. These investigators ${ }^{23}$ concluded that the marked reduction in whole body glucose transport was mainly due to a reduction in muscle mass, and that skeletal muscle had a remarkable capacity to maintain an intact glucose transport system despite denervation and severe morphological changes. Of note, Aksnes et $a l^{23}$ found that type IIb fibers were significantly increased from $29 \pm 2 \%$ in healthy controls to $66 \pm 6 \%$ in persons with tetraplegia. Using the euglycemic clamp technique, Lillioja ${ }^{24}$ demonstrated a significant correlation between insulin resistance and 
the percentage of type IIb muscle fibers, the dominant muscle fiber population after animal or human SCI. ${ }^{24,25}$ Type IIb muscle fibers are less sensitive to insulin-induced glucose uptake and have a reduced capillary density. In subjects with paraplegia, the contributions of innervated upper extremity or denervated lower extremity muscle to the insulin resistant state is unknown; selective glucose extraction across the arm or leg muscle beds during a hyperinsulinemic eugycemic clamp study should provide such information. ${ }^{26,27}$

An association between obesity and hyperuricemia in the able-bodied population has been reported. ${ }^{28}$ Zhong et $a l^{5}$ reported that in subjects with chronic SCI, the level of serum uric acid and serum triglycerides in the subgroup with hyperinsulinemia was significantly higher than in subjects with normal plasma insulin levels. The serum uric acid concentration was positively associated with peak plasma insulin level and BMI. ${ }^{5}$ In the study herein, serum uric acid was directly correlated to BMI, peak serum glucose and peak plasma insulin levels. Men, who were more insulin resistant (i.e., had higher plasma insulin levels for similar serum glucose concentrations), had a significantly higher serum uric acid concentration than women.

Of note, in a recent report in 541 subjects with SCI, the lowest serum high density lipoprotein (HDL) cholesterol levels were found in those with complete tetraplegia, and there was a graded increase of serum HDL cholesterol with decreasing neurological deficit. ${ }^{10}$ Although the serum triglycerides were not significantly different among the neurological subgroups, an inverse correlation was appreciated between HDL cholesterol and triglycerides. ${ }^{10}$

Since coronary heart disease has been reported to occur prematurely in persons with SCI, the question is whether this phenomenon is at least partially explicable on the basis of the metabolic milieu. Associations demonstrated among adiposity, carbohydrate intolerance, hyperinsulinism, adverse serum lipid values, and elevated serum uric acid suggest that individuals with SCI have several of the features of the metabolic constellation of Syndrome $\mathrm{X}^{3,29}$ and thus are at increased risk for coronary heart disease.

In conclusion, our findings suggest that degree of neurological deficit has an effect on carbohydrate tolerance. Individuals with the greatest neurological impairments have the worst carbohydrate tolerance and highest insulin values. In addition, being male adversely effects insulin sensitivity. Glucose tolerance appears to be independent of the effects of ethnicity. It remains to be determined as to whether pharmacological intervention or active exercise programs of the upper extremities and/or lower extremities by external electrical stimulation can prevent, forestall, or reverse the perturbations in carbohydrate and lipid metabolism in those individuals who manifest the highest risk for coronary heart disease.

\section{References}

1 Bauman WA, Spungen AM. Disorder of carbohydrate and lipid metabolism in veterans with paraplegia or quadriplegia: A model of premature aging. Metabolism 1994; 43: 749-756.

2 Reaven GM. NIDDM, abnormal lipoprotein metabolism, and atheroslcerosis. Metabolism 1987; 36 (Suppl. 1): 1-8.

3 DeFronzo RA, Ferrannini E. Insulin resistance. A multifaceted syndrome responsible for NIDDM, obesity, hypertension, dyslipidemia, and atherosclerotic cardiovascular disease. Diabetes Care 1991; 14: 173 - 194.

4 Bauman WA et al. Coronary artery disease: Metabolic risk factors and latent disease in individuals with paraplegia. Mount Sinai J Med 1992; 59: 163 - 168.

5 Zhong YG, Levy E, Bauman WA. The relationships among serum uric acid, plasma insulin, and serum lipoprotein levels in subjects with spinal cord injury. Horm Met Res 1995; 27: $282-$ 285.

6 Whiteneck G. Learning from empirical investigations. In: R Menter and G Whiteneck (eds). Perspectives on Aging with Spinal Cord Injury, Demos Publications. 1992, pp 23-37.

7 Yekutiel M et al. The prevalence of hypertension, ischemic heart disease and diabetes in traumatic spinal cord injured patients and amputees. Paraplegia 1989; 27: 58-62.

8 Geisler WO et al. Survival in traumatic spinal cord injury. Paraplegia 1983; 21: $364-373$.

9 Samsa P, Patrick C, Feussner JR. A national study of mortality among spinal cord injured veterans. IIR No. 87-036.

10 Bauman WA et al. The effect of residual neurological deficit on serum lipoproteins in individuals with chronic spinal cord injury. Spinal Cord 1998; 36: $13-17$.

11 American Spinal Injury Association. Standards for Neurological and Functional Classificiation of Spinal Cord Injury. Horm Met Res 1995; 27: $282-285$.

12 Berson SA, Yalow RS. Radioimmunoassay. In: Berson SA, Yalow RS (eds) Investigative and Diagnostic Endocrinology. North-Holland Publishing Co.: Amsterdam, The Netherlands. 1973, pp 84-120.

13 Yalow RS, Berson SA. General radioimmunoassay. In: Berson SA, Yalow RS (eds) Investigative and Diagnostic Endocrinology vol 23. North-Holland Publishing Co.: Amsterdan, The Netherlands. 1973, pp $864-870$.

14 The Expert Committee on the Diagnosis and Classification of Diabetes Mellitus. Report of the Expert Committee on the Diagnosis and Classification of Diabetes Mellitus. Diabetes Care 1997; 20: 1183 - 1197

15 Spungen AM, Bauman WA, Wang J, Pierson Jr, RN. Measurement of percent body fat in individuals with quadriplegia: a comparison of clinical methods. Paraplegia 1995; 33: $402-408$

16 Duckworth WC et al. Glucose intolerance due to insulin resistance in patients with spinal cord injuries. Diabetes 1980; 29: $906-910$.

17 WHO Expert Committee on Diabetes Mellitus: Second report. WHO Tech Rep Ser No 646, 1980.

18 Lipman RL et al. Impairment of peripheral glucose utilization in normal subjects by prolonged bed rest. J Lab Clin Med 1970; 76: $221-230$

19 Stern $\mathbf{J}$ et al. Adipose cell size and immunoreactive insulin levels in obese and normal weight adults. Lancet 1972; 2: $948-951$.

20 Olefsky JM. Decreased insulin binding to adipocytes and circulating monocytes from obese subjects. J Clin Invest 1976; 57: $1165-1172$.

21 Olefsky JM. The insulin receptor: Its role in insulin resistance of obesity and diabetes. Diabetes 1976; 25: $1154-1172$.

22 Bauman WA. Carbohydrate and lipid metabolism after spinal cord injury. Topics in Spinal Cord Injury Rehabilitation 1997; 2: $1-22$.

23 Aksnes AK et al. Intact glucose transport in morphologically altered denervated skeletal muscle from quadriplegic patients. Am J Physiol 1996; 271: E593 -E600. 
24 Lilloja $\mathrm{S}$ et al. Skeletal muscle capillary density and fiber type are possible determinants of in vivo insulin resistance in man. $J$ Clin Invest 1987; 80: $415-424$.

25 Grimby $\mathrm{G}$ et al. Muscle fiber composition in patients with traumatic cord lesion. Scand J Rehab Med 1976; 8: 37-42.

26 Andres R, Baltzan MA, Cader G, Zierler KL. Effect of insulin on carbohydrate metabolism and potassium in the forearm in man. J. Clin Invest 1962; 41: $108-115$.
27 Campbell PJ, Mandarino LJ, Gerish JE. Quantification of the relative impairment in actions of insulin on hepatic glucose production and peripheral glucose uptake in noninsulindependent diabetes mellitus. Metabolism 1988; 37: 15-21.

28 Acheson RM, Chan YK. New Haven survey of joint diseases. The prediction of serum uric acid in a general population. $J$ Chron Dis 1969; 21: $543-553$.

29 Reaven GM. Syndrome X. Clinical Diabetes 1994; 32 - 36. 